\title{
Polarization influences on the efficiency of noise gratings recorded in silver halide holograms
}

\author{
A. Beléndez, L. Carretero, and I. Pascual
}

\begin{abstract}
The efficiency of noise gratings recorded with single-beam exposures in bleached silver halide emulsions is analyzed as a function of the polarization state of the readout wave. Experimental results are presented and discussed on the basis of a theoretical model that considers both a linear and a nonlinear relation between the refractive-index modulation and the exposure. The dependence of the noise gratings' efficiency on the relative polarization between the construction and reconstruction beams is analyzed, and we find that this is represented by a quadratic curve of $\cos ^{2} \delta$, where $\delta$ is the polarization angle. The quantitative analysis is carried out with this model based on the coupled-wave theory, and the calculated results obtained by use of the theoretical model agree well with the experimental ones.

Key words: Bleached holograms, noise gratings, polarization.
\end{abstract}

\section{Introduction}

Silver halide emulsions are the most widely used recording material for making holographic optical elements because of their high sensitivity and ease of handling. However, a fundamental limitation of the holograms recorded in photographic emulsions is the scattering of light from the silver halide grains, ${ }^{1}$ which takes place both at the recording and at the reconstruction stages. During formation of a hologram, object fields scattered by the silver halide grains of the photographic emulsion interfere with the unscattered portion of the illuminating beam (which works as a reference beam), and because of this, spurious gratings are recorded in the medium. ${ }^{2}$ During the reconstruction stage of the hologram, these self-induced gratings (called noise gratings) diffract light in directions other than the direction of the signal beam, and this gives rise to a reduction in the diffraction efficiency and in the quality of the intentionally formed hologram.

Previous papers have reported the nature of these gratings and the effects of different parameters on the properties of noise gratings recorded in photographic emulsions. Noise gratings formed with a single beam and two beams of coherent monochromatic light have

The authors are with the Universidad de Alicante, Apartado 99, Alicante E 03080, Spain; A. Beléndez is with the Departamento de Ingeniería de Sistemas y Comunicaciones, and L. Carretero and I. Pascual are with the Departamento Interuniversitario de Optica. Received 9 February 1993. 0003-6935/93/357155-09\$06.00/0.

(C) 1993 Optical Society of America. been analyzed, ${ }^{2-7}$ and the characteristics of the noise gratings when the diffuse radiation is copied from a silver halide plate into thick dichromated gelatin have also been studied. ${ }^{8-10}$ Kostuk and Sincerbox ${ }^{11}$ reported the influence of the relative polarization between the recording and replay beams on these spurious gratings, although there were some errors in their theoretical analysis when they were obtaining the expression for the coupling coefficient for noise gratings. Also, as we noted in previous papers, ${ }^{12,13}$ noise gratings have a strong dependence on the emulsion processing chemistry, and it may be possible to reduce the efficiency of noise gratings by further optimization of the developer and the bleach bath. On the other hand, some theoretical models have been proposed in order to describe the characteristics of the noise gratings. A low-efficiency model ${ }^{14}$ has been developed, and experimental results agreed well with this theoretical model. By use of a statistical model it is possible to explain the behavioral changes in the efficiency of the noise gratings as a function of exposure, and a good agreement between the theoretical and experimental results has been found. ${ }^{15}$

The aim of the present paper is to extend further the studies cited in Ref. 11. We present experimental results and a theoretical analysis of the influence of the polarization angle (defined as the angle between the polarization vectors of the construction and reconstruction beams) on the diffraction efficiency of noise gratings recorded in bleached silver halide holograms. The replay characteristics of these gratings are analyzed at various replay polarizations, 
and the corresponding efficiencies are calculated. The quantitative analysis of the effect of polarization on noise gratings is carried out with the coupled-wave theory, as reported in Ref. 11. Experiments are performed by recording of holograms with singlebeam exposures in Agfa 8E75HD plates, and the results obtained agree well with the theory.

\section{Formation of Noise Gratings}

Noise gratings were formed by a single collimated beam polarized perpendicular to the plane of incidence, and the beam was produced by a $15-\mathrm{mW}$ $\mathrm{He}-\mathrm{Ne}$ laser at a wavelength of $632.8 \mathrm{~nm}$ in Agfa Gevaert 8E75HD photographic emulsion. Singlebeam exposures were made with the illuminating beam entering normal to the surface of the emulsion. Eight holograms were made, and the exposure levels used were $40,50,75,100,150,200,300$, and 400 $\mu \mathrm{J} / \mathrm{cm}^{2}$. Spurious reflections were eliminated by placement of an index-matched absorbing layer against the glass side of the photographic plate. After exposure, the plates were developed in AAC developer, which consists of a solution of ascorbic acid $(18 \mathrm{~g})$ and sodium carbonate $(60 \mathrm{~g})$ in $1 \mathrm{~L}$ of distilled water. After a brief rinse, the plates were bleached without a fixation step with an R-10 rehalogenatingtype bleach. ${ }^{16}$ This bath was composed of potassium dichromate $(2 \mathrm{~g})$, sulfuric acid $(10 \mathrm{~mL})$, and potassium bromide $(35 \mathrm{~g})$ diluted in $1 \mathrm{~L}$ of distilled water. This processing technique is known to introduce small changes in the emulsion thickness ${ }^{16,17}$ and the average refractive index, ${ }^{17}$ and hence it may be expected to enhance the efficiency of noise gratings. Then, when reconstruction and recording angles coincide, it is possible to satisfy Bragg's Law simultaneously for every elementary grating of the noise grating. ${ }^{2,3}$

On the other hand, we obtained the HurterDriffield curve $(D-\log E)$, where $D$ is the density and $E$ is the exposure, for Agfa 8E75HD holographic emulsion with a $632.8-\mathrm{nm}$ coherent monochromatic light and the AAC developer. By observing the plot of this curve, we deduced that the exposures made between 40 and $100 \mu \mathrm{J} / \mathrm{cm}^{2}$ were practically in the linear region of this curve, while the rest of the exposure levels used belonged to the saturation region of the $D-\log E$ curve. This fact indicates that for exposures between 40 and $100 \mu \mathrm{J} / \mathrm{cm}^{2}$ the response of the holographic material is linear and the refractive-index modulation varies linearly with exposure. However, as the exposure is increased, the nonlinearities in the emulsion response also increase, and the index change therefore is not proportional to exposure. ${ }^{18}$

\section{Measurements}

The holograms were replayed in air with a collimated beam of coherernt monochromatic light at $632.8 \mathrm{~nm}$. The transmittance of each plate was measured as a function of the replay angle, ${ }^{2}$ and plots such as those shown in Fig. 1 were obtained. In this figure the pronounced drop in transmission at the construction

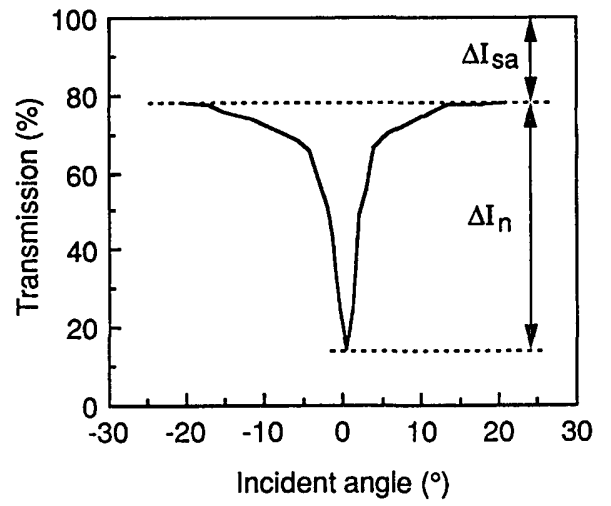

Fig. 1. Typical plot of transmittance as a function of the reconstruction angle for noise gratings recorded with a single beam of light.

angle $\left(0^{\circ}\right)$ is caused by the presence of noise gratings. On the other hand, $\Delta I_{a s}$ corresponds to the combined average absorption and scattering, ${ }^{13}$ and $\Delta I_{n}$ represents the efficiency of the noise gratings. The value of $\Delta I_{n}$ indicates an increase in scattering above the average scattering and absorption levels for a replay angle equal to the construction angle; this angle is the Bragg angle for the noise gratings formed in the emulsion.

The plane defined by the emulsion surface normal and the electric field of the recording beam was used as a reference for the polarizations of both the recording and the replay beams. We replayed the holograms with a reconstruction beam entering normal to the plate but polarized in a direction different to the polarization of the recording beam. We define a polarization angle $\delta$ so that $\delta=0^{\circ}$ for the polarization of the reconstruction beam parallel to the polarization of the construction beam and $\delta=90^{\circ}$ when polarizations of the reconstruction and construction beams are perpendicular. In order to investigate the influence of the $\delta$ angle on the efficiency of the noise gratings, we measured the transmission of the plates for five values of the polarization angle $\delta: 0^{\circ}, 30^{\circ}$, $45^{\circ}, 60^{\circ}$, and $90^{\circ}$. We calculated the efficiency of the noise gratings, $\Delta I_{n}$. The error in the efficiency of these gratings was $\sim 5 \%$. Figure 2 shows the values of $\Delta I_{n}$ as a function of exposure for the polarization angles considered. As we can see from this figure, the noise-grating efficiency decreases when angle $\delta$ increases, and $\Delta I_{n}$ is a function of exposure.

In order to obtain experimentally an empirical relation between noise-grating efficiency and the polarization angle, we plotted $\Delta I_{n}$ as a function of $\delta$, cos $\delta$, and $\cos ^{2} \delta$, and we observed that the best plot obtained corresponded to the graph of $\Delta I_{n}$ versus $\cos ^{2}$ $\delta$. Figure 3 shows the measured diffraction efficiency of the noise gratings as a function of $\cos ^{2} \delta$ for holograms exposed with different total energies $E$. In this figure the curves show the adjustment of the experimental data by a quadratic curve and use of a least-squares method. In all situations analyzed the corresponding regression coefficients were between 0.99 and 1.00 . This fact indicates that the calculated 


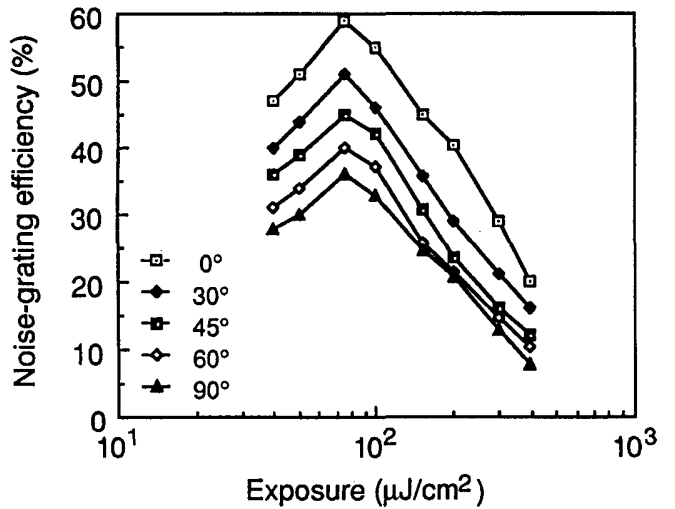

Fig. 2. Noise-grating efficiency as a function of the incident exposures for polarization angles $\delta$ equal to $0^{\circ}, 30^{\circ}, 45^{\circ}, 60^{\circ}$, and $90^{\circ}$.

data with an empirical function in the form

$$
\Delta I_{n}(\delta)=a_{1}+a_{2} \cos ^{2} \delta+a_{3} \cos ^{4} \delta
$$

agreed well with the experimental results. In Eq. (1) the $a_{i} s$ coefficients were a function of exposure. On the other hand, for exposure levels between 40 and $100 \mu \mathrm{J} / \mathrm{cm}^{2}$ (linear region of the $D-\log E$ curve) we can observe from Fig. 3 that the adjusted quadratic curves have approximately the same shape. For these exposures the normalized empirical curves $\Delta I_{n}(\delta) / \Delta I_{n 0^{\circ}}\left[\right.$ where $\left.\Delta I_{n 0^{\circ}}=\Delta I_{n}\left(0^{\circ}\right)\right]$ practically coin-
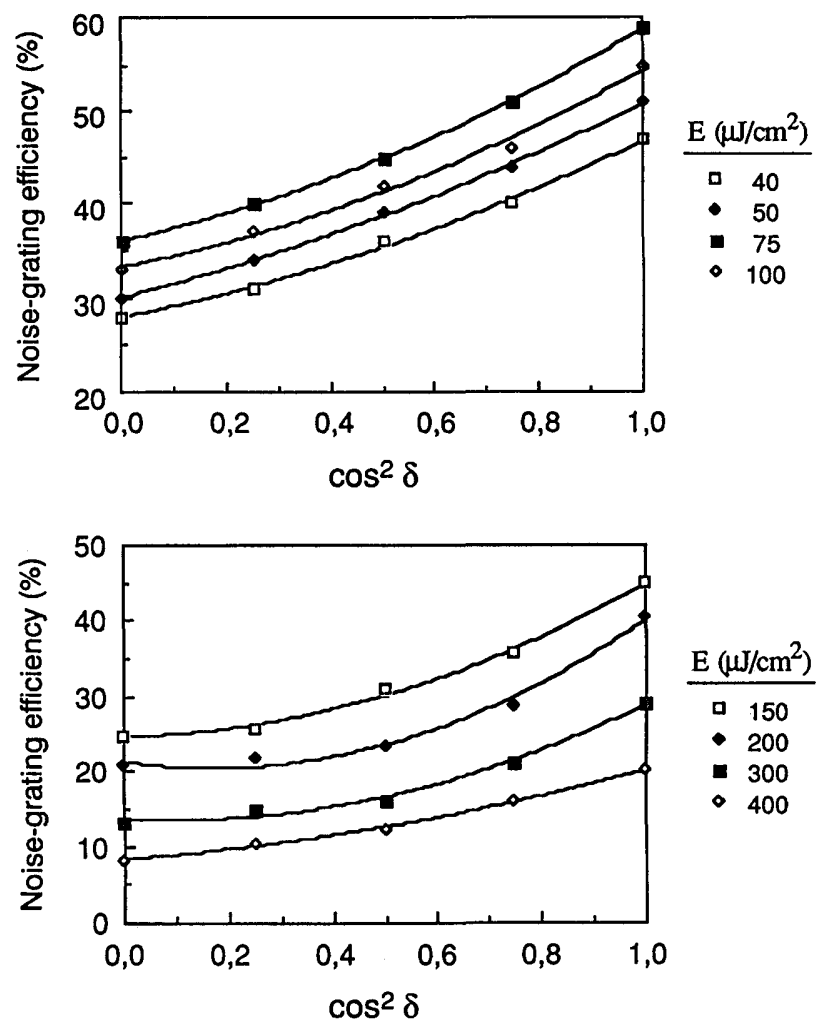

Fig. 3. Measured diffraction efficiency of the noise gratings as a function of $\cos ^{2} \delta$ for holograms exposed with different total energies $E$. Solid curves show the adjustments of the experimental data by a quadratic curve. cide, and they do not depend upon exposure. However, for exposures in the saturation region of the D-log E curve the normalized curves $\Delta I_{n}(\delta) / \Delta I_{n 0^{\circ}}$ do not coincide.

\section{Theoretical Analysis}

In this section a simple method for describing the experimental results of Section 3 is presented. The model proposed is based on the analysis made by Kostuk and Sincerbox, ${ }^{11}$ and it takes into account the light-scattering properties of the silver halide emulsion. A photographic emulsion consists of a gelatinous emulsion of approximately $5-7-\mu \mathrm{m}$ thickness deposited on a plastic foil or on a glass backing. Finegrain silver halide clusters $\sim 35 \mathrm{~nm}$ in diameter are suspended in the gelatin-based matrix and sensitized by doping with heavy metal atoms. Since the $\mathrm{Ag} \mathrm{Br}$ grains are irregular in shape and randomly distributed, the polarizability of the medium can be assumed to be isotropic. As the ratio of the average grain size to the illumination wavelength is $<7 \%$, we consider that the Rayleigh scattering predominates.

When an illumination plane wave of coherent monochromatic light of wavelength $\lambda$ incides on the photographic plate, a dipole moment $\mathbf{p}=\alpha \mathbf{E}_{\mathrm{r}}$ is induced on each silver halide grain, where $\alpha$ is the electrical polarizability of the silver halide grain and $\mathbf{E}_{r}$ is the electric field of the illumination beam. If $\mathbf{e}_{r}$ is the unit vector in the direction of polarization of the illumination beam (we name this the reference beam), we can write $\mathbf{E}_{r}$ as $\mathbf{E}_{r}=E_{r} \mathbf{e}_{r}$. Figure 4 shows the geometry to be analyzed. The $z$ axis is chosen parallel to vectors $\mathbf{e}_{r}$ and $\mathbf{p}$. The scattered radiation is in all possible directions with amplitude a function of direction. The resulting electric field $\mathbf{E}_{s}$ of the scattered beam at some point at a distance $r$ from the dipole and in the $(\theta, \phi)$ direction has the form

$$
\mathbf{E}_{s}=E_{s}(\theta) \exp \left(j \psi_{s}\right) \mathbf{e}_{s} .
$$

In this equation $\mathbf{e}_{s}$ is the unit vector in the direction of polarization of the scattered beam, $E_{s}(\theta)=E_{s} \sin \theta$, $E_{s}=k^{2} p / r$, and $k=2 \pi / \lambda$. The intensity of the light scattered by a silver halide grain is proportional to the square of $E_{s}(\theta)$. Figure 5 shows the intensity profile ${ }^{9}$ both in a plane perpendicular [Fig. 5(a)] and parallel [Fig. 5(b)] to the dipole vector p. In Figs. 4 and 5, $\mathbf{k}_{r}$ and $\mathbf{k}_{s}$ are the propagation vectors of the reference and scattered beams, respectively. As we can see from Fig. 5, intensity is a function of the $\theta$ angle (for $\theta=0^{\circ}$ the intensity is null, and for $\theta=90^{\circ}$ the intensity is maximum); however, this scattering intensity is not a function of the $\phi$ angle.

Next we consider the interference pattern produced by the reference wave that was incident upon the photographic emulsion and the wave diffused (object wave) by an emulsion particle after the reference wave has been incident upon the emulsion particle. Following the Kostuk treatment, we obtain irradi- 


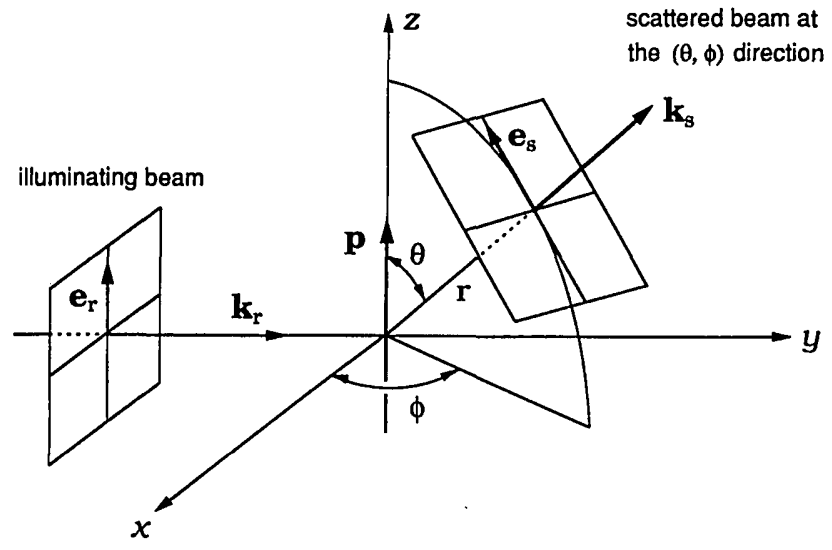

Fig. 4. Geometry for analysis of dipole scattering. $\mathbf{p}$ is the dipolar moment induced in each silver halide grain.

ance $I_{r s}$ in the recording material, given by ${ }^{11}$

$$
\begin{aligned}
I_{r s} & =\left|\mathbf{E}_{r}+\mathbf{E}_{s}\right|^{2}=\left|E_{r} \exp \left(j \psi_{r}\right) \mathbf{e}_{r}+E_{s}(\theta) \exp \left(j \psi_{s}\right) \mathbf{e}_{s}\right|^{2} \\
& =\left|E_{r}\right|^{2}+\left|E_{s}(\theta)\right|^{2}+2 E_{r} E_{s} \cos \psi_{r s} \sin \theta\left(\mathbf{e}_{r} \cdot \mathbf{e}_{s}\right),
\end{aligned}
$$

where $\psi_{r s}=\psi_{r}-\psi_{s}$. We also accept as an additional hypothesis that the holographic recording material is processed in such a way that we end up with a phase grating. Thus the exposure distribution of the fringes is converted to the spatial variation of the refractive index $\Delta n$ of the hologram through processing. Assuming that the variation of the refractive index is linearly proportional to the exposure, we can write $^{11}$

$$
\Delta n \propto E_{r} E_{s} \sin \theta\left(\mathbf{e}_{r} \cdot \mathbf{e}_{s}\right) \propto \sin \theta\left(\mathbf{e}_{r} \cdot \mathbf{e}_{s}\right) .
$$

$\Delta n$ depends on the $\theta$ angle, but it is not a function of the $\phi$ angle.

The diffraction efficiency $\eta$ of the reconstructed field is proportional to the square of the coupling coefficient ${ }^{11} \kappa$, which, for a pure phase grating, is also proportional to the refractive index modulation $\Delta n$ and the scalar product of the unit polarization vectors of the reconstruction $\left(\mathbf{e}_{c}\right)$ and diffracted $\left(\mathbf{e}_{d}\right)$ beams:

$$
\kappa \propto \Delta n\left(\mathbf{e}_{c} \cdot \mathbf{e}_{d}\right) .
$$

Then, the diffraction efficiency is

$$
\eta(\theta, \phi, \delta) \propto \kappa^{2} \propto \Delta n^{2}\left(\mathbf{e}_{c} \cdot \mathbf{e}_{d}\right)^{2},
$$
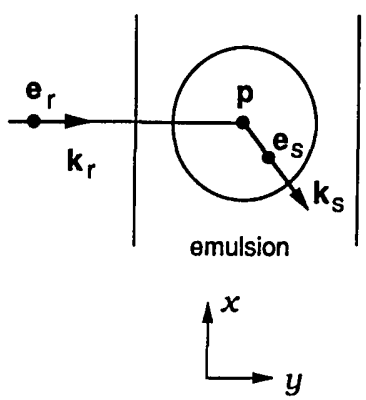

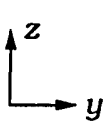

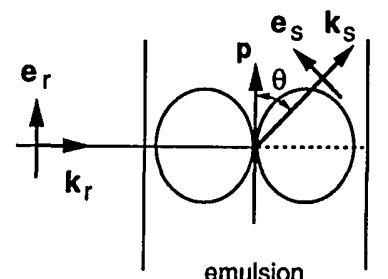

Fig. 5. Intensity profile (from Ref. 9) in a plane (a) perpendicular and (b) parallel to the dipole vector $\mathbf{p}$. and taking into account relation (4), we can write

$$
\eta(\theta, \phi, \delta) \propto\left(\mathbf{e}_{r} \cdot \mathbf{e}_{s}\right)^{2}\left(\mathbf{e}_{c} \cdot \mathbf{e}_{d}\right)^{2} \sin ^{2} \theta,
$$

where we write explicitly that the efficiency is a function of the $(\theta, \phi)$ direction of the diffracted beam and the polarization angle ( $\delta$ ). From Fig. 4 the scalar product of the unit polarization vectors $\mathbf{e}_{r}$ and $\mathbf{e}_{s}$ is $\sin \theta$, and then

$$
\eta(\theta, \phi, \delta) \propto\left(\mathbf{e}_{c} \cdot \mathbf{e}_{d}\right)^{2} \sin ^{4} \theta .
$$

If the reconstructed beam enters normal to the surface of the plate (as does the reference beam) and the angle between the polarization vectors of the reconstruction $\left(\mathbf{e}_{c}\right)$ and reference $\left(\mathbf{e}_{r}\right)$ beams is $\delta$ (polarization angle), we simplify the problem by assuming that for a replay beam entering normal to the surface of the plate the polarization light in each direction $(\theta, \phi)$ is simply the same as the corresponding recording polarization with a constant added rotation angle $(\delta)$ when the replay beam polarization is rotated by the $\delta$ angle. This can be seen by comparison of Figs. 4 and 6, and this approximation is based on the experimental results for diffraction efficiency that we have obtained by using planar transmission gratings recorded and replayed with collimated beams at normal incidence and reconstructed with different polarization angles $(\delta)$. From Fig. 6 we can write

$$
\begin{aligned}
& \mathbf{u}_{\theta}=\cos \phi \cos \theta \mathbf{u}_{x}+\sin \phi \cos \theta \mathbf{u}_{y}-\sin \theta \mathbf{u}_{z}, \\
& \mathbf{u}_{\phi}=-\sin \phi \mathbf{u}_{x}+\cos \phi \mathbf{u}_{y}, \\
& \mathbf{e}_{c}=\sin \delta \mathbf{u}_{x}+\cos \delta \mathbf{u}_{z}, \\
& \mathbf{e}_{d}=-\cos \delta \mathbf{u}_{\theta}-\sin d \mathbf{u}_{\phi},
\end{aligned}
$$

where $\mathbf{u}_{x}, \mathbf{u}_{y}$, and $\mathbf{u}_{z}$ are the unit vectors in the directions of the $x, y$, and $z$ axes, respectively. From Eqs. (9)-(12) the scalar product $\mathbf{e}_{c} \cdot \mathbf{e}_{d}$ is given by

$$
\begin{aligned}
\mathbf{e}_{c} \cdot \mathbf{e}_{d}= & \sin \theta \cos ^{2} \delta+\sin \phi \sin ^{2} \delta \\
& -\cos \theta \cos \phi \sin \delta \cos \delta .
\end{aligned}
$$

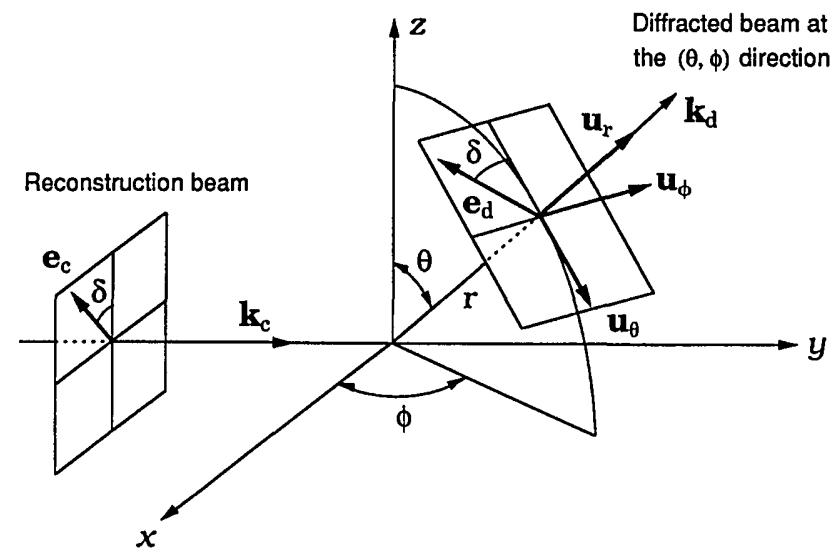

Fig. 6. Geometry for analysis of the reconstruction of the noise gratings. $\delta$ is the angle between the $\mathbf{e}_{r}$ and $\mathbf{e}_{c}$ vectors. 
And the diffraction efficiency in the direction defined by angles $\theta$ and $\phi$ and for a polarization angle $\delta$ has the form

$$
\begin{aligned}
\eta(\theta, \phi, \delta) \propto & \sin ^{6} \theta \cos ^{4} \delta+\sin ^{4} \theta \sin ^{2} \phi \sin ^{4} \delta \\
& +2 \sin ^{5} \theta \sin \phi \sin ^{2} \delta \cos ^{2} \delta \\
& +\sin ^{4} \theta \cos ^{2} \theta \cos ^{2} \phi \sin ^{2} \delta \cos ^{2} \delta \\
& -2 \sin ^{4} \theta \cos \theta \cos \phi \sin \phi \sin ^{3} \delta \cos \delta \\
& -2 \sin ^{5} \theta \cos \theta \cos \phi \sin \delta \cos ^{3} \delta .
\end{aligned}
$$

The diffraction efficiency for all possible diffracted beams, $\eta(\delta)$, can be calculated through the integral of the efficiencies $\eta(\theta, \phi, \delta)$ in the individual beam for all possible directions characterized by angles $\theta$ and $\phi$, and it has the form

$$
\eta(\delta) \propto 2 \int_{0}^{\pi} d \phi \int_{0}^{\pi} d \theta \sin \theta \eta(\theta, \phi, \delta) .
$$

In this relation we have accepted as an additional hypothesis that the reflected scattered radiation does not play a significant role. ${ }^{8,13}$ If we consider that the total diffraction efficiency $\Delta I_{n}(\delta)$ of all gratings from the random ensemble of dipole scatters is proportional to the efficiency of a single dipole scatter $\eta(\delta)$, we can write

$$
\Delta I_{n}(\delta) \propto \int_{0}^{\pi} \mathrm{d} \phi \int_{0}^{\pi} \mathrm{d} \theta \sin \theta \eta(\theta, \phi, \delta) .
$$

It is convenient to normalize this efficiency with the efficiency $\Delta I_{n 0^{\circ}}=\Delta I_{n}\left(\delta=0^{\circ}\right)$ when the directions of polarization for the construction and reconstruction beams are the same $\left(\delta=0^{\circ}\right)$. This normalized efficiency takes the form

$$
\frac{\Delta I_{n}(\delta)}{\Delta I_{n 0^{\circ}}}=\frac{\int_{0}^{\pi} \mathrm{d} \phi \int_{0}^{\pi} \mathrm{d} \theta \sin \theta \eta(\theta, \phi, \delta)}{\int_{0}^{\pi} \mathrm{d} \phi \int_{0}^{\pi} \mathrm{d} \theta \sin \theta \eta\left(\theta, \phi, 0^{\circ}\right)} .
$$

Finally, by use of Eq. (14) the following relation can be obtained:

$$
\begin{aligned}
\frac{\Delta I_{n}(\delta)}{\Delta I_{n 0^{\circ}}} & =\frac{1}{384}\left(224+109 \cos ^{2} \delta+51 \cos ^{4} \delta\right) \\
& \approx 0.583+0.284 \cos ^{2} \delta+0.133 \cos ^{4} \delta
\end{aligned}
$$

This expression is not a function of the incident exposure and gives us the normalized diffraction efficiency as a function of polarization angle $\delta$ for a linear recording material. As we can see from this relation, there is the same dependence in $\cos ^{2} \delta$ as in the experimental equation obtained in Section 3 [Eq. (1)].

Another important parameter that we define for characterizing the influence of the polarization angle on noise-grating efficiency is the quotient between the efficiencies for polarization angles $\delta=90^{\circ}$ (minimum efficiency of $\Delta I_{n 90^{\circ}}$ ) and $\delta=0^{\circ}$ (maximum efficiency of $\left.\Delta I_{n 0^{\circ}}\right)$. From expression (18) we can obtain

$$
\frac{\Delta I_{n 90^{\circ}}}{\Delta I_{n 0^{\circ}}}=\frac{7}{12} \approx 0.583
$$

Expression (18) was obtained by consideration that there exists a linear dependence between the refractive-index modulation and exposure in the form $\Delta n \propto$ $E_{r} E_{s} \sin \theta\left(\mathbf{e}_{r} \cdot \mathbf{e}_{s}\right)=E_{r} E_{s} \sin ^{2} \theta$. However, as we pointed out in Section 2, when exposure increases, nonlinearities in the emulsion response appear, and the relation between $\Delta n$ and exposure is not linear. We suppose that the amplitude of each scattered wave $E_{s}$ is proportional to the incident wave amplitude $E_{s}$ according to the expression ${ }^{15} E_{s}=E_{r} / K^{1 / 2}$, where $K$ is the beam ratio between the incident irradiance $\left|\mathbf{E}_{r}\right|^{2}$ and the irradiance of the scattered wave $\left|\mathbf{E}_{s}\right|^{2}$. Taking this into account, we see that $\Delta n$ is a function of $E_{r} E_{s} \sin \theta\left(\mathbf{e}_{r} \cdot \mathbf{e}_{s}\right)=E_{r} E_{s} \sin ^{2} \theta \propto E \sin ^{2} \theta$, where $E=\left|\mathbf{E}_{r}\right|^{2}$ is the incident exposure. For the nonlinear region we suppose that $\Delta n$ is represented by a function so that

$$
\Delta n=\Delta n_{0}\left[1-\exp \left(-E \sin ^{2} \theta / E_{0}\right)\right],
$$

where $\Delta n_{0}$ and $E_{0}$ are constants. We can write $\Delta n$ approximately in the form

$$
\Delta n \approx \Delta n_{0}\left[\left(E / E_{0}\right) \sin ^{2} \theta-(1 / 2)\left(E / E_{0}\right)^{2} \sin ^{4} \theta\right] .
$$

Expression (21) indicates that the refractive-index modulation $\Delta n$ can be represented by a polynomial of up to the second order of $E \sin ^{2} \theta$. The square of $\Delta n$ is approximately

$$
\begin{aligned}
\Delta n^{2} \approx & \Delta n_{0}^{2}\left(E / E_{0}\right)^{2}\left[\sin ^{4} \theta-\left(E / E_{0}\right) \sin ^{6} \theta\right. \\
& \left.+(1 / 4)\left(E / E_{0}\right)^{2} \sin ^{8} \theta\right] .
\end{aligned}
$$

Taking into account the expression obtained for $\Delta n^{2}$, relation (6), and Eq. (17), we obtain the following expression for the normalized efficiency of the noise gratings:

$$
\frac{\Delta I_{n}(\delta)}{\Delta I_{n 0^{\circ}}}=\frac{1}{B}\left(A_{1}+A_{2} \cos ^{2} \delta+A_{3} \cos ^{4} \delta\right),
$$

where

$$
\begin{gathered}
A_{1}=3.351-2.872\left(E / E_{0}\right)+0.638\left(E / E_{0}\right)^{2}, \\
A_{2}=1.631-1.447\left(E / E_{0}\right)+0.328\left(E / E_{0}\right)^{2}, \\
A_{3}=0.763-0.787\left(E / E_{0}\right)+1.161\left(E / E_{0}\right)^{2}, \\
B=5.745-5.106\left(E / E_{0}\right)+2.127\left(E / E_{0}\right)^{2} .
\end{gathered}
$$

In Eqs. (24)-(27), $E$ is the total exposure of the 
incident beam and $E_{0}$ is a constant whose value is calculated by fitting of the experimental results to the theoretical ones. From Eq. (23), when $E \rightarrow 0$, we obtain expression (18), which corresponds to the linear region. For the nonlinear analysis parameter, $\Delta I_{n 90^{\circ}} / \Delta I_{n 0^{\circ}}$ (minimum value of the normalized efficiency) takes the form

$$
\frac{\Delta I_{n 90^{\circ}}}{\Delta I_{n 0^{\circ}}}=\frac{3.351-2.872\left(E / E_{0}\right)+0.638\left(E / E_{0}\right)^{2}}{5.745-5.106\left(E / E_{0}\right)+2.127\left(E / E_{0}\right)^{2}} .
$$

As we can see from this equataion, the quotient $\Delta I_{n 90^{\circ}} / \Delta I_{n 0^{\circ}}$ does not have a fixed value as in the linear case but depends on the incident exposure $E$. For $E \rightarrow 0$, Eq. (28) coincides with expression (19).

The normalized diffraction efficiency $\Delta I_{n}(\delta) / \Delta I_{n 0^{\circ}}$ of the noise gratings [Eq. (23)] is plotted in Fig. 7 as a function of $\cos ^{2} \delta$ for $E_{0}=340 \mu \mathrm{J} / \mathrm{cm}^{2}$ and various values of the incident exposure: $40,200,300$, and $400 \mu \mathrm{J} / \mathrm{cm}^{2}$. The curve for $E=40 \mu \mathrm{J} / \mathrm{cm}^{2}$ coincides with the curve calculated by use of expression (18) that is obtained when a linear relation between $\Delta n$ and $E$ is used. As we can see in Fig. 7, the minimum value of the normalized efficiency (the quotient $\left.\Delta I_{n 90^{\circ}} / \Delta I_{n 0^{\circ}}\right)$ decreases with an increase in exposure $E$.

\section{Comparison between Experimentation and Theory}

In this section we show the comparison between the experimental results and the theoretical analysis,

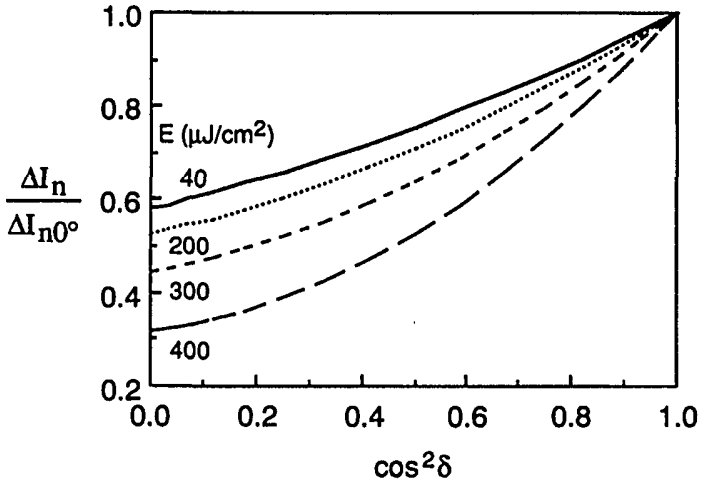

Fig. 7. Normalized diffraction efficiency $\Delta I_{n}(\delta) / \Delta I_{n 0^{\circ}}$ of the noise gratings as a function of $\cos ^{2} \delta$ for various values of the incident exposure $E$ when the effect of nonlinearities is taken into account.

shown in Sections 3 and 4, respectively. Figures 8 and 9 show the experimental data and the theoretical curves for the normalized diffraction efficiency of the noise gratings $\left(\Delta I_{n} / \Delta I_{n 0^{\circ}}\right)$ as a function of $\cos ^{2} \delta$ for different values of the exposure of the recording beam. Theoretical curves are shown by solid and dashed curves, and the experimental data are shown by solid squares. The solid curve in each figure corresponds to the theoretical curve calculated by consideration of a linear relation between $\Delta n$ and exposure expression (18)], while the dashed curve was calculated by use of Eq. (23) and the value of 340
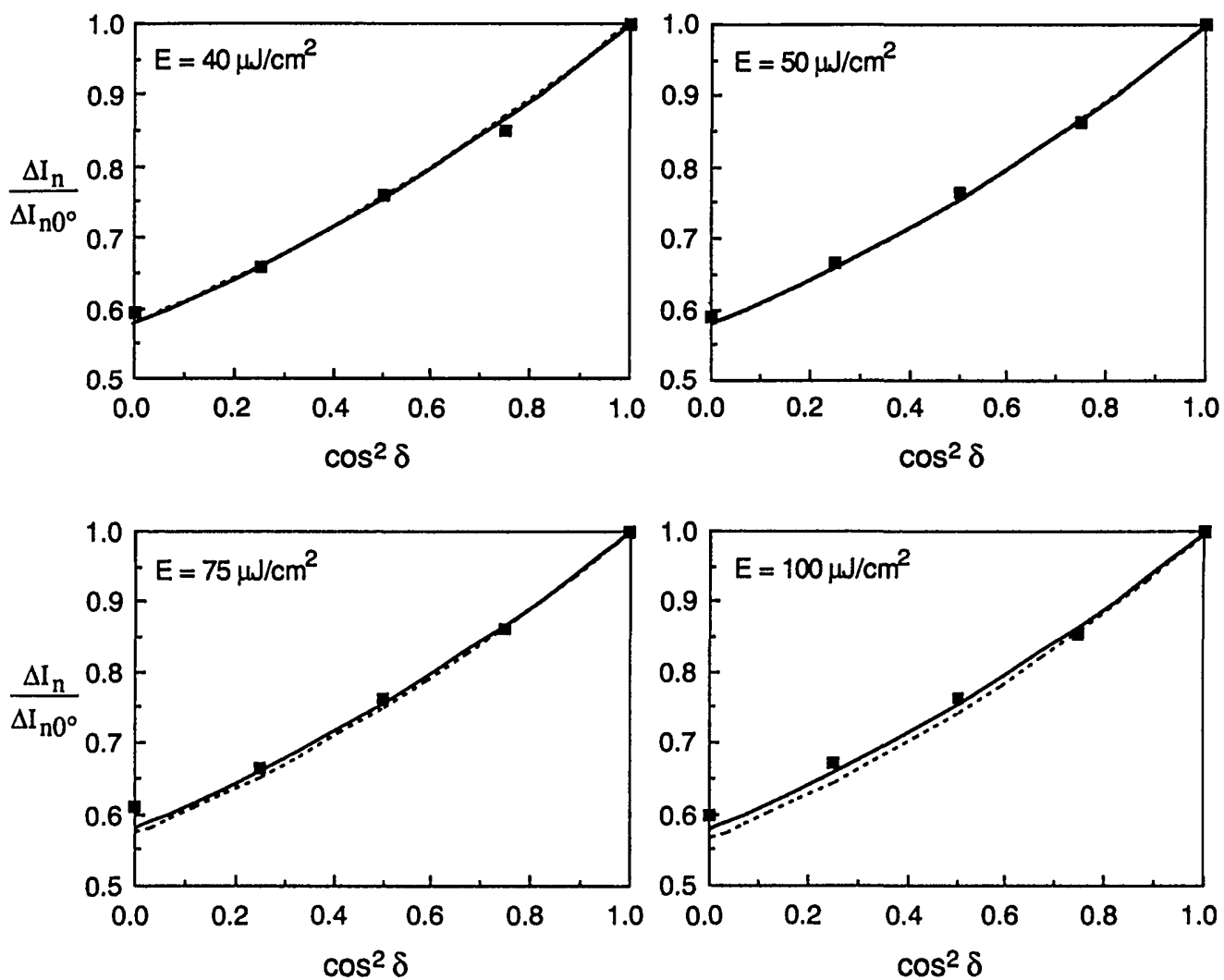

Fig. 8. Experimental data $(\mathbb{a})$ and theoretical curves for the normalized efficiency of noise gratings $\Delta I_{n}(\delta) / \Delta I_{n 0^{\circ}}$ as a function of $\cos ^{2} \delta$ for low exposures. In each graph the solid curve corresponds to the theoretical curve calculated assuming a linear relation between $\Delta n$ and exposure, and each dashed curve is calculated with the effect of nonlinearities taken into account. 

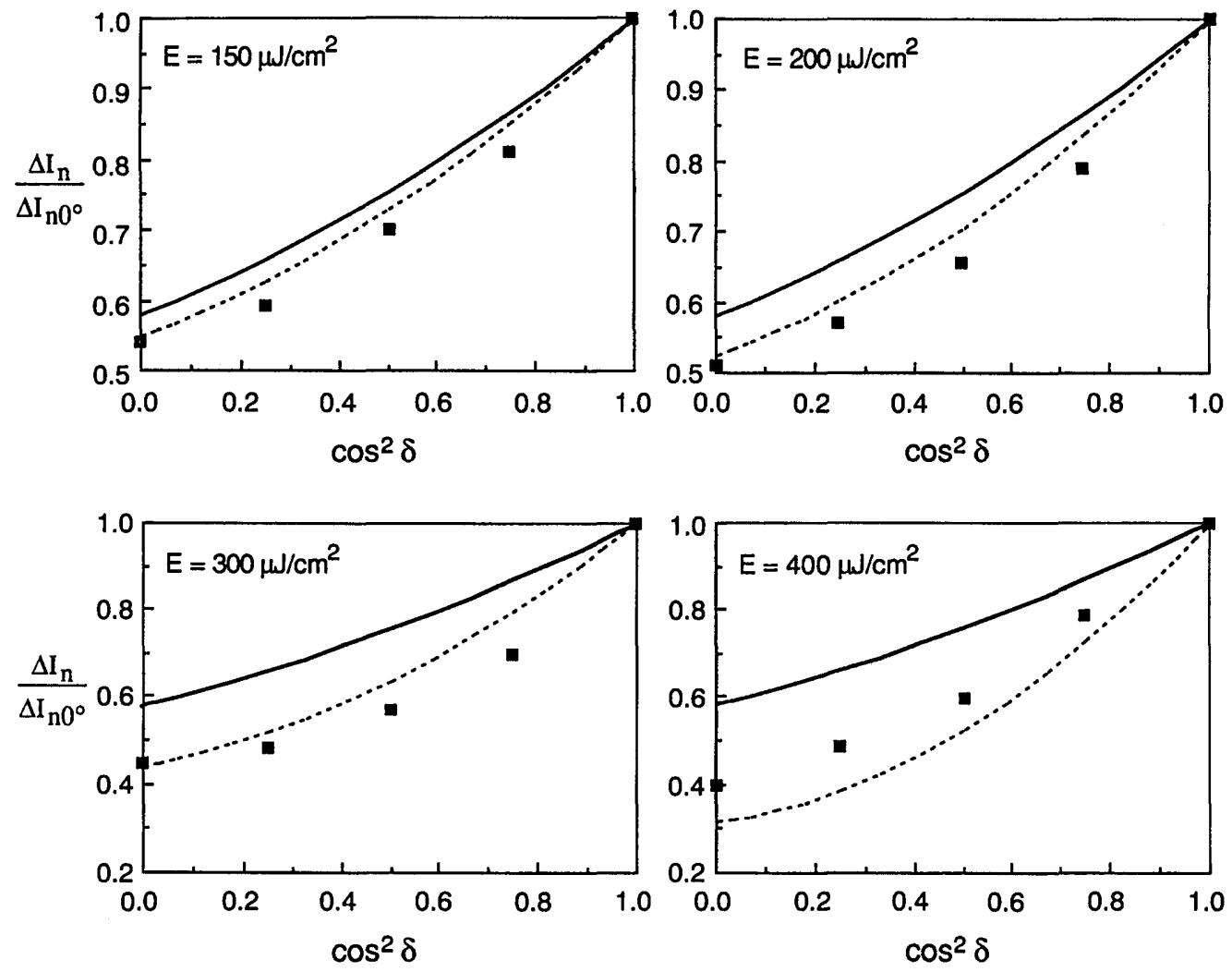

Fig. 9. Same as Fig. 8 but for high exposures.

$\mu \mathrm{J} / \mathrm{cm}^{2}$ for parameter $E_{0}$ (the nonlinear relation between $\Delta n$ and exposure). The value for $E_{0}=340$ $\mu \mathrm{J} / \mathrm{cm}^{2}$ was obtained by fitting of the experimental data to the theoretical values. As can be seen from these figures, the two theoretical curves coincide for low exposures (Fig. 8), and the theoretical data agree very well with the experimental results for this range of exposures. However, for high exposures (Fig. 9) there are some differences between the two theoretical curves, and these differences increase with exposure. For these last levels of exposure there is good agreement between the predicted results obtained through Eq. (23) (in which we considered a nonlinear relation between $\Delta n$ and exposure) and the measured data.

Figure 10 shows the experimental and theoretical data for parameter $\Delta I_{n 90^{\circ}} / \Delta I_{n 0^{\circ}}$ (the minimum value of the normalized efficiency) as a function of the incident exposure. The dashed curve corresponds to the values for this parameter when it is assumed that $\Delta n$ is proportional to exposure [expression (19)], and then $\Delta I_{n 90^{\circ}} / \Delta I_{n 0^{\circ}}$ is constant. In Fig. 10 the solid line was obtained by consideration of nonlinear effects and with Eq. (28). As can be seen from this figure the agreement between experimental and theoretical data is very good for all exposures when nonlinearities are taken into account. However, the measured and the theoretical data calculated under the linear approximation coincide only for low exposures.
Finally, by use of the theoretical model developed in this paper it is possible to explain an experimental observation reported in Kostuk's paper. ${ }^{11}$ They observed the scattered light of holograms (recorded with a single beam of coherent light) in a plane parallel to the holographic plate and situated at a specific distance from the plate. They concluded that the preferred scattering direction in this observation plane was perpendicular to the polarization direction when

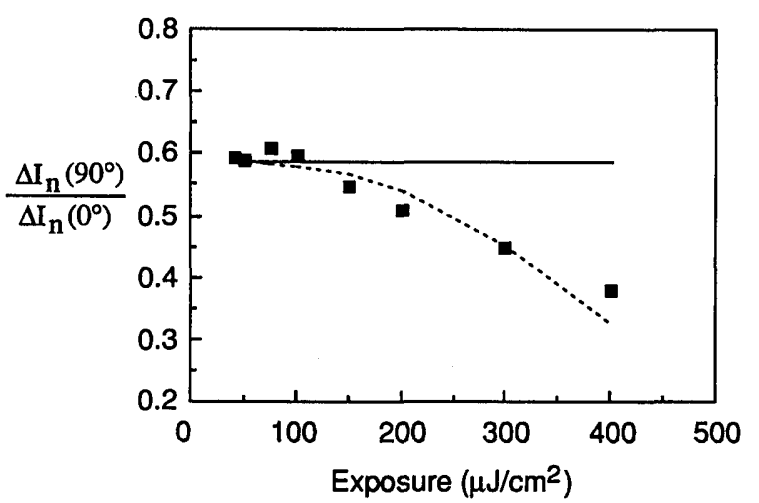

Fig. 10. Experimental and theoretical data for parameter $\Delta I_{n 90^{\circ}} / \Delta I_{n 0^{\circ}}$ as a function of the incident exposure. The solid line corresponds to the values obtained when $\Delta n$ is proportional to exposure, and the dashed curve was obtained assuming a nonlinear relation between $\Delta n$ and exposure. 

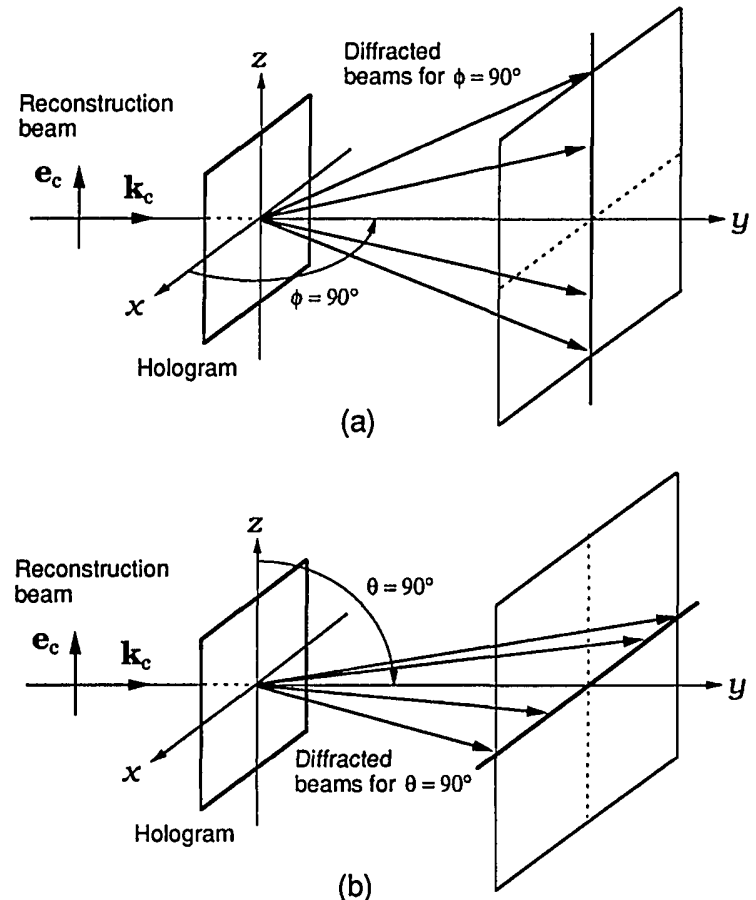

Fig. 11. Scheme for analyzing the influence of the direction of observation in a plane parallel to the hologram plane on noisegrafting efficiency $\Delta I_{n}$ : (a) direction parallel and (b) perpendicular to the polarization of the reconstruction beam.

the directions of polarization for the recording and readout beams were the same. Figure 11 shows a scheme that can be used for analysis of these experimental results theoretically. The direction of the unit polarization vectors of the recording and replay beams, $\mathbf{e}_{r}$ and $\mathbf{e}_{c}$, coincides $\left(\delta=0^{\circ}\right)$, and it is parallel to the $z$ axis. First, we calculate the efficiency of the noise gratings for $\delta=0^{\circ}$. From relation (14) we can write

$$
\eta\left(\theta, \phi, \delta=0^{\circ}\right) \propto \sin ^{6} \theta .
$$

If $\eta\left(\theta, \phi=90^{\circ}, \delta=0^{\circ}\right)$ is the noise-grating efficiency for different values of angle $\theta$ and a fixed value of angle $\phi=90^{\circ}$ [see Fig. 11(a)], it is possible to obtain a noise-grating efficiency $\Delta I_{n}\left(\phi=90^{\circ}, \delta=0^{\circ}\right)$ in a plane perpendicular to the hologram and in a direction parallel to the polarization direction ( $z$ axis), as follows:

$$
\begin{aligned}
\Delta I_{n}\left(\phi=90^{\circ}, \delta=0^{\circ}\right) & \propto \int_{0}^{\pi} \eta\left(\theta, \phi=90^{\circ}, \delta=0^{\circ}\right) \sin \theta \mathrm{d} \theta \\
& =\int_{0}^{\pi} \sin ^{7} \theta \mathrm{d} \theta=\frac{32}{35}
\end{aligned}
$$

On the other hand, the noise-grating efficiency for different values of angle $\phi$ and a fixed value of angle $\theta=90^{\circ}$ [see Fig. $\left.11(\mathrm{~b})\right]$ is $\eta\left(\theta=90^{\circ}, \phi, \delta=0^{\circ}\right) \approx 1$.
Now $\Delta I_{n}\left(\theta=90^{\circ}, \delta=0^{\circ}\right)$ is the noise-grating efficiency in a plane parallel to the hologram and in a direction perpendicular to the polarization direction ( $x$ axis), and it is expressed as

$$
\begin{aligned}
& \Delta I_{n}\left(\phi=90^{\circ}, \delta=0^{\circ}\right) \\
& \quad \propto \int_{0}^{\pi} \eta\left(\theta=90^{\circ}, \phi, \delta=0^{\circ}\right) \mathrm{d} \phi=\int_{0}^{\pi} \mathrm{d} \phi=\pi .
\end{aligned}
$$

From relations (30) and (31) it is possible to obtain

$$
\frac{\Delta I_{n}\left(\theta=90^{\circ}, \delta=0^{\circ}\right)}{\Delta I_{n}\left(\phi=90^{\circ}, \delta=0^{\circ}\right)}=\frac{\pi}{32 / 35} \approx 3.44
$$

and then

$$
\Delta I_{n}\left(\theta=90^{\circ}, \delta=0^{\circ}\right)>\Delta I_{n}\left(\phi=90^{\circ}, \delta=0^{\circ}\right) .
$$

This inequality indicates that for $\delta=0^{\circ}$ the efficiency in the direction perpendicular to the direction of the polarization of the beams [Fig. 11(b)] is higher than that in the direction parallel to the polarization of the beams [Fig. 11(a)]. This is in agreement with the aforementioned experimental results.

We can also obtain an expression similar to inequality (33) for polarization states such as $\delta=90^{\circ}$ (when unit polarization vectors $\mathbf{e}_{r}$ and $\mathbf{e}_{c}$ are perpendicular). Following a procedure similar to the one used previously, we can easily obtain the inequality

$$
\Delta I_{n}\left(\theta=90^{\circ}, \delta=90^{\circ}\right)>\Delta I_{n}\left(\phi=90^{\circ}, \delta=90^{\circ}\right) .
$$

Now the preferred scattering direction is parallel to the direction of polarization of the replay beam, which is perpendicular to the direction of polarization of the recording beam.

\section{Conclusions}

The experimental results and the theoretical analysis shown in this paper indicate that the efficiency of the spurious noise gratings recorded in bleached silver halide emulsions depends largely on the relation between the polarization states of the recording and readout beams. A theoretical model has been developed that considers both linear and nonlinear relations between the refractive-index modulation and the exposure of the incident beam. The model predicts that the efficiency of the noise gratings depends on polarization angle $\delta$. It was also shown that for a reconstruction polarization orthogonal to the recording polarization $\left(\delta=90^{\circ}\right)$ the model predicts that noise grating efficiency is less than for parallel polarizations $\left(\delta=0^{\circ}\right)$ but that it is not zero (as Kostuk and Sincerbox mentioned in their paper ${ }^{11}$ ), and this result is confirmed by the experiments. Good agreement between experiment and theory was obtained for the range of exposures analyzed, although this agreement is better for low exposures. The theoretical curves obtained had the same shape as the experimen- 
tal plots, and there was also agreement between the measured and the calculated values of the minimum normalized efficiency. Finally the analysis of the influence of polarization on the noise-grating efficiency described in this paper gives us more information about the mechanism of formation and the characteristics of these spurious gratings generated by scattering during recording.

The authors thank A. Fimia for his interest in this work and for fruitful discussions. They also express their sincere thanks to the referees for their valuable suggestions.

\section{References}

1. K. Biedermann, "The scattered flux spectrum of photographic materials for holography," Optik 31, 367-389 (1970).

2. R. R. A. Syms and L. Solymar, "Noise gratings in photographic emulsions," Opt. Commun. 43, 107-110 (1982).

3. R. R. A. Syms and L. Solymar, "Noise gratings in silver halide volume holograms," Appl. Phys. B 30, 177-182 (1983).

4. A. A. Ward, J. M. Heaton, and L. Solymar, "Efficient noise gratings in silver halide emulsions," Opt. Quantum Electron. 16, 365-367 (1984).

5. L. Solymar and G. D. G. Riddy, "Noise gratings for single- and double-beam exposures in silver halide emulsions," J. Opt. Soc. Am. A 7, 2107-2108 (1990).

6. L. Wang and R. K. Kostuk, "Direct formation of planar holograms and noise gratings at $820 \mathrm{~nm}$ in bleached silver halide emulsions," Opt. Lett. 14, 919-921 (1989).

7. G. D. G. Riddy, "The effect of light scattering on the recording and replay of holographic optical elements," Ph.D. dissertation (Oxford University, Oxford, 1988).

8. L. Solymar and J. C. W. Newell, "Silver halide noise gratings recorded in dichromated gelatin," Opt. Commun. 73, 273-276 (1989).

9. L. T. Blair and L. Solymar, "Angular selectivity of silver halide transmission noise gratings copied into dichromated gelatin," Appl. Opt. 29, 2985-2986 (1990).

10. L. T. Blair and L. Solymar, "Silver halide reflection noise gratings recorded in dichromated gelatin," Opt. Commun. 77, 126-128 (1990).

11. R. K. Kostuk and G. T. Sincerbox, "Polarization sensitivity of noise gratings recorded in silver halide volume holograms," Appl. Opt. 27, 2993 -2998 (1988).

12. A. Beléndez, I. Pascual, and A. Fimia, "Noise gratings recorded with single-beam exposures in silver halide emulsions: the influence of the bleach bath," Opt. Quantum Electron. 25, 139-145 (1993).

13. L. Carretero, A. Beléndez, and A. Fimia, "Holographic noise gratings for analyzing and optimizing photochemical processings in bleached silver halide emulsions," J. Mod. Opt. 40, 687-697 (1993).

14. G. D. G. Riddy and L. Solymar, "Theoretical model of reconstructed scatter in volume holograms," Electron. Lett. 22, 872-873 (1986).

15. L. Carretero, A. Fimia, and A. Beléndez, "Statistical model for noise gratings recorded in volume holograms," J. Mod. Opt. 40, 1299-1308 (1993).

16. J. Crespo, A. Fimia, and J. A. Quintana, "Fixation-free methods in bleached reflection holography," Appl. Opt. 25, 1642-1645 (1986).

17. R. K. Kostuk, "Factorial optimization of bleach constituents for silver halide holograms," Appl. Opt. 30, 1611-1616 (1991).

18. C. W. Slinger, R. R. A. Syms, and L. Solymar, "Nonlinear recording in silver halide planar volume holograms," Appl. Phys. B 36, 217-224 (1985). 